\title{
Financing Patient Education in Arthritis and Musculoskeletal and Skin Diseases
}

\author{
Jean Goeppinger and Jane Walter
}

On March 23-24, 1989, a conference, Financing Patient Education in Arthritis and Musculoskeletal and Skin Diseases, was held in Atlanta, Georgia. This conference was sponsored by the National Arthritis Advisory Board (NAAB). It recognized the significant progress that has been made in establishing the effectiveness of arthritis patient education since the first NAAB-sponsored conference, which focused on patient education, was held in 1980 (Arthritis Research and Education in Nursing and Allied Health) and supported today's needs to establish the cost-effectiveness of arthritis patient education and secure adequate reimbursement for structured educational efforts. Speakers and participants reflected the diverse constituencies of patient education: health education researchers; health care professionals, including physicians, health educators, nurses, and allied health professionals; representatives of federal and private health insurance organizations; representatives of the Arthritis Foundation and the National Psoriasis Foundation; members of federal advisory bodies such as the National Arthritis Advisory Board; persons with arthritis, musculoskeletal, and skin diseases; and social and behavioral scientists.

The opening presentations, by Drs. Halsted Holman ("Why Patient Education?"), Steven Maz-

Jean Goeppinger, $\mathrm{PhD}, \mathrm{RN}$ is Professor and Chair of Community Health Nursing at the University of Michigan School of Nursing. Jane Walter, PT, EdD is Associate Professor, Division of Physical Therapy, Division of Clinical Immunology and Rheumatology at the University of Alabama at Birmingham.

Address all correspondence to Jean Goeppinger, PhD, RN, University of Michigan School of Nursing, $400 \mathrm{~N}$. Ingalls, Ann Arbor, MI 48109.

(C) 1989 by the Arthritis Health Professions Association zuca ("Does Patient Education in Chronic Disease Have Therapeutic Value?"), and Lawren Daltroy ("Critical Summary of the Field"), built a convincing argument for the effectiveness of arthritis patient education in improving commonly accepted clinical outcome measures, e.g., pain, dysfunction and disability, and depression. Dr. Kate Lorig ("Does Patient Education Require a Prescription?") added another perspective to the argument, that of health education as a right, a consumer-driven health care demand rather than a physician-controlled component of medical treatment.

The opening speakers were followed by panel discussions of the barriers to reimbursement existing within (1) Federal health programs, e.g., the prospective payment and resource-based relative value systems, (2) Federally funded health insurance programs such as Medicare, and (3) the private insurance industry. Representatives from the private sector stressed that health insurance most readily reimburses for random, unexpected events rather than the predictable, persistent problems associated with chronic disease (Louis Sapparito) and that the efficacy of patient education must be firmly established and clearly articulated (Dr. Halley Faust).

In conclusion, a series of case studies were presented that documented successful arthritis education efforts in a variety of settings despite the barriers. Janice Smith-Pigg described an ambulatory comprehensive arthritis program in a private hospital. Patient education is provided as part of this program and is, to some extent, reimbursed. Dr. Wendy Cuneo described how the equality of patient education was assured in a large health maintenance organization. Suzanne Koebel and Marsha Dubbert described state-supported arthritis programs in Ohio and Missouri, respectively. Nina Berlin outlined an action model for securing reimbursement, based on 
successful experiences in diabetes patient education.

The remainder of this summary highlights the major issues identified at the conference and concludes with a set of recommendations for action developed at two post-conference sessions, one chaired by Dr. Sam Brown and organized by the NAAB and the other organized and chaired by Dr. John Winfield for the American College of Rheumatology. Representatives of the Arthritis Health Professions Association, the Arthritis Foundation, the National Arthritis Advisory Board, and some conference participants attended.

\section{THE ISSUES}

Although the effectiveness of arthritis patient education in improving commonly accepted clinical measures has been rather consistently demonstrated, several issues remain to be addressed before the argument is sufficiently convincing to permit reimbursement for patient education. These issues include the lack of evidence regarding the effect of patient education on health services utilization. Dr. Holman and his research colleagues at Stanford have demonstrated that 4 years after 233 elderly persons took the Arthritis Self-Help course, $34 \%$ had fewer visits to the physician than prior to course participation. Attempts to replicate these findings have not been made, however, and, more importantly, the essential cost-benefit and cost-effectiveness studies have not been conducted.

Another issue raised is that few studies have demonstrated the extent to which the findings from relatively well-controlled research experiments can be replicated in generally uncontrolled service settings. Dissemination efforts have occurred but have not been carefully monitored; process and outcome evaluations of the service-based replications of experimental research protocols need to be conducted. Such studies are not highly valued by the scientific community and are not well funded; they are, however, essential to building the case for reimbursement.

Three, the effects of patient education efforts in musculoskeletal and skin diseases other than arthritis have not been examined; the work done in patient education of persons with osteoarthritis, rheumatoid arthritis, and low back pain, however, can be instructive (Daltroy). A set of clinical parameters must be identified as acceptable outcome measures; patient education interventions need to be developed; carefully controlled research trials need to be conducted; and cost-benefit and cost-effectiveness studies need to be planned.

Four, the impact of reimbursing professionally led patient education interventions on volunteer, mutual aid approaches to health education must be considered so that disincentives are not unintentionally created (Barbara Giloth). The relative contributions of health education that is patient-oriented and professionally provided and health education that is community-based and lay-led must be considered.

Finally, we must consider the issue of whether patient education is simply a physician's duty, to be reimbursed accordingly, or whether structured health education is to be offered and reimbursed as part of a multidisciplinary approach to the care of persons with arthritis, musculoskeletal, and skin diseases.

The first four issues can be resolved largely through the continued development, testing, dissemination, and evaluation of education interventions for persons with arthritis, musculoskeletal, and skin diseases. The last issue, on the other hand, suggests that plans must be developed to ensure the consistent provision and quality of education interventions and to obtain third-party reimbursement. Work on these issues can proceed concurrently, with researchers assuming major responsibility for advancing the science of patient/health education and clinicians, patients, and their advocates, and voluntary organizations assuming responsibility for influencing policy development. The remainder of the meeting and the postconference sessions emphasized steps necessary to the development of adequate financing mechanisms.

Dr. Cuneo stated that to engage in quality assurance efforts, we need to know the following: what is most important to the patient education program and to the sponsoring organization; the acceptable indicators, outcomes, and/or standards of successful patient education; the extent to which variability around the standards will be tolerated; how to incorporate peer review; and how to document and report findings. Ms. Berlin illustrated such activities in the effort she led on behalf of the National Diabetes Advisory Board to secure reimbursement for diabetes patient education that was initiated in the late 1970s. The National Diabetes Advisory Board began by developing consensus papers on the treatment of five complications of diabetes and identifying standards for acceptable diabetes education programs. She stressed the importance of becoming acquainted with the health care financing system(s) and the locus of decision-making; working with, not legislat- 
ing against, third-party insurers; learning to market education programs; and learning to negotiate with insurers on a case-by-case basis.

\section{RECOMMENDATIONS}

These comments guided participants in the postconference sessions to develop the following recommendations for actions designed to secure reimbursement for arthritis patient education:

1. Designate a representative national organization or coalition to assume leadership in developing reimbursement for arthritis patient education.

2. Establish standards for structured arthritis patient education programs.

3. Evaluate the cost-effectiveness of arthritis patient education.

4. Obtain financial reimbursement by successful marketing and negotiation with representatives of health insurance organizations and federal and state health policymakers.

On April 17, 1989, the NAAB agreed to act as the catalyst for these efforts. The Board plans to propose a process for developing standards for patient education in arthritis, musculoskeletal, and skin diseases. The proposal will be discussed, modified, and approved at a NAAB-initiated meeting of representatives from relevant professional and voluntary organizations and successful state-wide programs. Responsibility for implementing the process of standard development will then be assumed by participating organizations; the NAAB will continue to be involved in a coordinating role. The NAAB intends that this process will ensure that progress achieved during the 1970s and 1980s will be utilized in the 1990s to secure improved financing for patient education in arthritis, musculoskeletal, and skin diseases. 